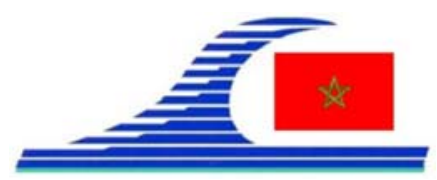

Conférence Méditerranéenne Côtière et Maritime EDITION 2, TANGER, MAROC (2011)

Coastal and Maritime Mediterranean Conference

Disponible en ligne - http://www.paralia.fr - Available online

\title{
L’apport de la géomatique dans la gestion des zones côtières. Application d'un SIG côtier (Rif nord occidental, Maroc)
}

\author{
Rabii EL HAMZAOUI ${ }^{1}$, Ahmed RAISSOUNI ${ }^{1}$, Abdelkrim EL ARRIM ${ }^{1}$
}

1. Université Abdelmalek Essaâdi, Faculté des Sciences et Techniques, Département des Sciences de la Terre, Laboratoire "Environnement, Océanologie et Ressources Naturelles" (LEORN), Equipe de Recherche en Environnement Marin et Risques Naturels (EREMRN), BP: 416, Tanger, Maroc.

elhamzaoui.rabii@gmail.com ; ah_raissouni@hotmail.com ; elarrimk@yahoo.fr

\section{Résumé :}

Le Maroc possède un vaste territoire maritime. Au Nord, les côtes méditerranéennes qui s'étendent sur 512 kilomètres, connaissent une dégradation progressive entraînant, par endroits, un recul important de la ligne des côtes, surtout le tronçon littoral entre Tanger-Tétouan.

Afin d'améliorer la gestion stratégique de la défense des littoraux pour un développement durable des zones côtières du Nord du pays, la mise en œuvre d'un Système d’Information Géographique Côtier (SiCoast) a pour objectif la gestion intégrée et durable du domaine maritime public "onshore" et "offshore".

Le système SiCoast sera conçu pour recueillir des informations alphanumériques et géographiques sur de nombreux aspects (géomorphologie ; nature ; paysage ; domaines public, administratif et réglementaire) des municipalités côtières.

Ainsi, face au développement de la technologie, l'utilisation de la géomatique avec surtout l'apport des données satellitaires de moyenne et haute résolution et les systèmes d'information géographique, s’impose pour une gestion plus efficace des zones côtières.

\section{Mots-clés :}

Littoral nord occidental - SiCoast - Géomatique

\section{Introduction}

La zone côtière est un espace extrêmement complexe dont le fonctionnement et l'évolution sont conditionnés par de multiples paramètres naturels et anthropiques qui interagissent à différentes échelles spatiotemporelles (GOURMELON \& ROBIN, 2005).

Le littoral marocain, doté de deux façades maritimes, l'une atlantique qui s'étend de Tanger à Lagouira (2934 km) et l'autre méditerranéenne de Tanger à Saïdia (512km), constitue un pôle d'attraction important pour les activités socio-économiques (exploitations touristiques, installations industrielles et portuaires, pêche, ...) et abrite un patrimoine environnemental varié, riche et vulnérable (faune et flore, paysages et sites naturels tels que les dunes et les zones humides, etc.). Ainsi cet espace hautement 
La connaissance de la Mer :

un vecteur du développement durable en Méditerranée

attractif pour la concentration humaine et urbanistique, connait des problèmes tels que l'érosion côtière, la pollution, la dégradation des dunes, inondations par les eaux marines, l'intrusion saline dans les aquifères côtiers et les rivières, ensablement de l'arrière côte, .... .

Dans un tel contexte, l'appréhension de ces problèmes s'avère indispensable et incontournable dans la problématique du développement durable qui vise à préserver l'environnement. A cet égard, de nouvelles visions et de nouvelles technologies modernes ont été impliqués, il s'agit principalement de la Gestion Intégrée des Zones Côtières (GIZC) et l'utilisation des technologies spatiales et de plus particulièrement les Systèmes d'Information Géographique et la télédétection spatiale, afin d'améliorer les processus décisionnels et la gestion transversale et multi-actionnaire dans ces zones côtiers.

\section{Présentation de la zone d'étude}

Située à l'extrême Nord Ouest du Royaume du Maroc, la région de Tanger-Tétouan est limitée au Nord par le Détroit de Gibraltar et la Méditerranée, à l'Ouest par l'Océan Atlantique, au Sud par la région du Gharb et à l'Est par la région de Taza - Al Hoceima - Taounate.

S'étendant sur une superficie de $11570 \mathrm{~km}^{2}$, soit 1,6\% de la superficie totale du pays, la région s'inscrit dans le domaine du climat méditerranéen et est constituée de quatre unités environnementales homogènes : le Tangérois, la côte et les bassins méditerranéens, Jbala et le Loukkos.

Elle se compose actuellement de deux préfectures et trois provinces; il s'agit des préfectures de Tanger-Assilah et Fahs-Bni Makada et des provinces de Chefchaouen, Larache et Tétouan. En outre, la région englobe 10 cercles, 87 communes rurales et 13 municipalités.

Sa population, qui est de 2,04 millions d'habitants en 1994, soit $8 \%$ de la population totale du pays, a atteint 2,8 millions d'habitants en 2010 dont 86\% en milieu urbain. Eu égard à sa superficie, la région est considérée comme l'une des régions les plus peuplées du Maroc et les plus densifiées (176 habitants $/ \mathrm{km}^{2}$ en 1994 contre 37 au niveau du pays).

\section{Matériels et méthodes}

La complexité des zones côtières rend leurs gestion et sauvegarde difficiles. Elles requièrent une information de bonne qualité et actualisée pour aider à prendre les bonnes décisions. Ceci donne une importance particulière à la gestion de l'information dans la prise de décision sur les zones côtières (NAKHLI \& GHAZI, 2008).

Les Systèmes d'Information Géographique s'inscrivent dans un ensemble d'approches, de méthodes et d’outils désigné par le terme générique de "Géomatique". Si l’intérêt des SIG pour l'environnement littoral est reconnu depuis plusieurs décennies, c’est 
seulement à partir du début des années 1990 que les applications se sont diversifiées et multipliées (GOURMELON \& ROBIN, 2005).

La mise en œuvre d'un Système d'Information Géographique Côtier (SiCoast), surtout avec l'apport des données satellitaires de moyenne et haute résolution, a pour objectif la gestion intégrée et durable du domaine maritime public "onshore" et "offshore".

Le système SiCoast sera conçu pour recueillir des informations alphanumériques et géographiques sur de nombreux aspects (géomorphologie, nature, paysage, domaines public, administratif et réglementaire) des municipalités côtières.

La méthodologie de travail s'articule autour de 4 grandes phases :

- Phase 1 : Collecte de données géographiques et documentaires qui concernent le domaine côtier (images satellitaires, photographies aériennes, levés GPS, démographique, administrative, économique, sociale et commerciale, etc.) ;

- Phase 2 : Organisation, normalisation et analyse des données ;

- Phase 3 : Création d'une base de données géographiques composée de tableaux contenant des données alphanumériques et géographiques ;

- Phase 4 : Développement d'une application web qui utilise des technologies libres pour la consultation du système.

Cette approche méthodologique sera accompagnée par une analyse de durabilité visant à produire une liste d'indicateurs de durabilité-clés, indispensables au suivi et à l'évaluation de la pertinence de la gestion intégrée de la zone côtière du Rif nord occidental. Actuellement, les indicateurs visés couvrent deux des quatre piliers du développement durable, à savoir la composante environnementale ; le recul du trait de côte, et la composante socio-culturelle ; le linéaire côtier urbanisé.

\section{Résultats}

Les résultats attendus (EL HAMZAOUI, 2010) seront la mise en œuvre d'un système d'information géographique côtier conçu pour recueillir des informations sur de nombreux aspects (géomorphologie, nature, paysage, administratif, réglementaire) des municipalités côtières, permettant ainsi la visualisation et interrogation des données cartographiques et les informations alphanumériques liées, utiles pour une planification et une gestion intégrée des zones côtières (figure 1).

Le système regroupe les fonctionnalités de base d'un Système d'Information Géographique : navigation, requête attributaire, requête spatiale, outil de dessin, outil de mesure, impression et export, ce qui permettra la mise à jour continuelle des données.

L'interface WEB du SiCoast vise les utilisateurs par la visualisation interactive de l'ensemble des couches de données, à savoir l'occupation du sol, le transport, la ville, etc. acquises sur la zone littorale.

La gestion de l'interface et de sa base de données est assurée par un serveur principal, ayant comme fonction la réception, la gestion, et l'émission des données recueillis des différents participants au réseau. 
La connaissance de la Mer :

un vecteur du développement durable en Méditerranée

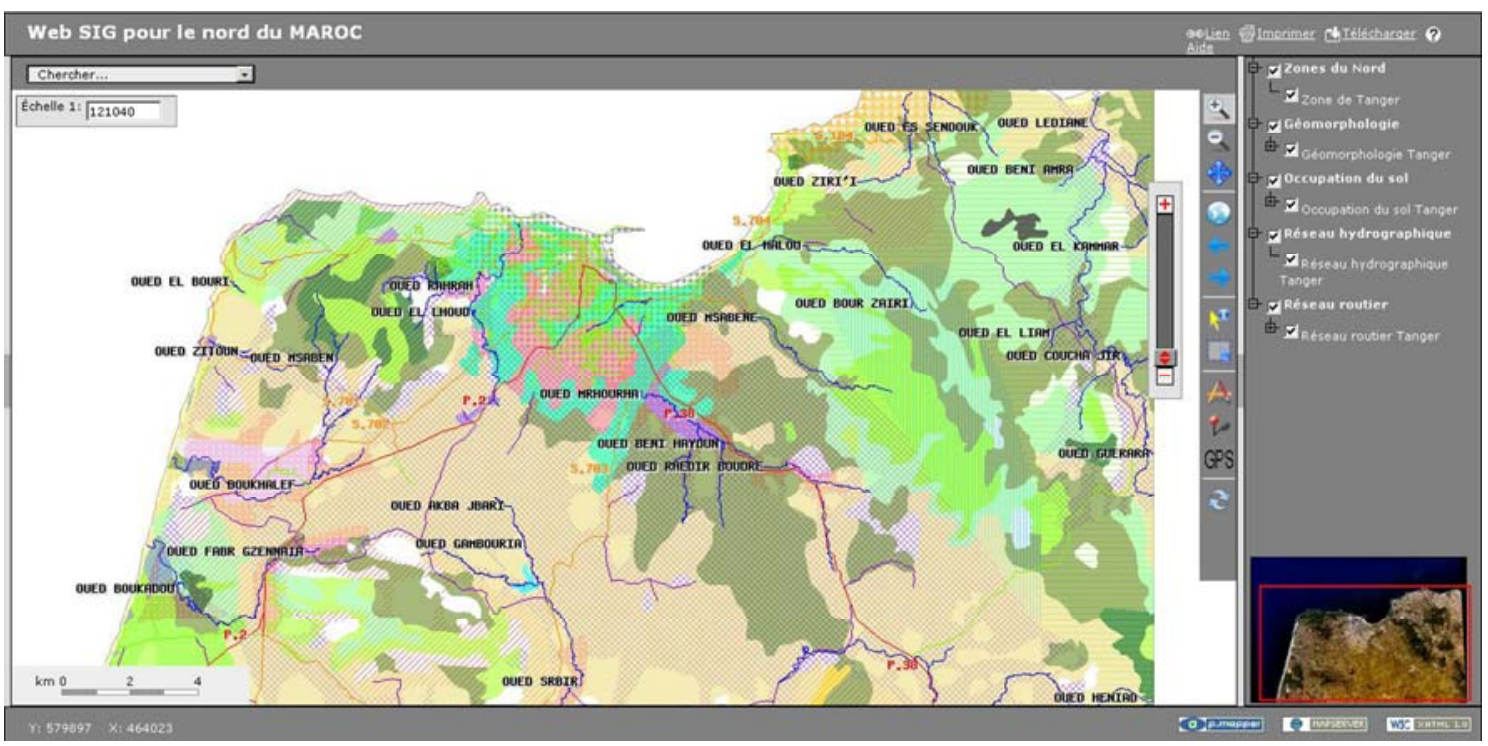

Figure 1. Interface de SiCoast.

\section{Conclusion}

La zone côtière est un espace extrêmement complexe dont le fonctionnement et l'évolution sont conditionnés par de multiples paramètres naturels, physique et anthropiques qui interagissent à différentes échelles spatiotemporelles. Une telle complexité ne peut se gérer qu’à travers des outils adaptés, capables de prendre en compte l'espace et le temps. Les SIG sont ces outils nécessaires pour stocker, mémoriser, traiter puis diffuser les différentes informations disponibles afin de faire émerger une représentation plus synthétique et utilisable plus facilement pour les décisions au service des différents acteurs du développement durable des zones côtières. La mise en place du système SiCoast serait alors un outil utile, en mesure de transférer son expérience à l'échelle de la région Rif nord occidental, permettant de légitimer une démarche dynamique, prospective, systémique et interdisciplinaire pour la GIZC.

\section{Références bibliographiques}

EL HAMZAOUI R. (2010). Développement d'un prototype web de gestion des données alphanumériques d'un SIG côtier (SiCoast) de la région de Ligurie (NW. Italie). Application du système sur la zone Nord du Maroc, 67 p.

GOURMELON F., ROBIN M. (2005). SIG et littoral. Hermès, Paris, 328 p.

NAKHLI S., GHAZI A. (2008). Quels outils pour un développement durable des zones côtières marocaines. Actes du Coll. Int. "Le littoral : subir, dire, agir", Lille, France, pp 1-7. [disponibe sur : http://www.meshs.fr/documents/pdf/publications/actes/colloque_littoral/Nakhli-Ghazi.pdf ] 